\title{
Estudio de la vascularización arterial del músculo flexor digitorum superficialis
}

\section{Anatomical study of the arterial vascularization of the flexor digitorum superficialis muscle}

\author{
Comellas Melero, N. *, Salvador-Sanz, J .F.**, Terol Calpena, F. ***, Almenar García, V. ***, \\ Pérez Cano, R. ${ }^{* * * *}$
}

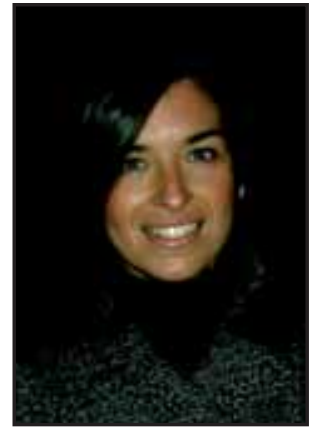

Comellas Melero, $\mathrm{N}$.

\section{Resumen}

El objetivo de nuestro estudio es describir la distribución de la vascularización del músculo flexor digitorum superficialis para optimizar sus indicaciones en Cirugía Reconstructiva de miembro superior. Disecamos 15 antebrazos fijados según el método Thiel y coloreados mediante la inyección de látex en los vasos femorales. Centramos nuestro estudio en la disección del músculo flexor digitorum superficialis, seleccionando únicamente los pedículos vasculares que superan los $2 \mathrm{~mm}$, valorando los resultados en función de sus relaciones anatómicas y de su longitud.

El número total hallado de arterias nutrientes del vientre muscular fue de 219 , localizándose en mayor porcentaje en el tercio medio del antebrazo.

De los resultados obtenidos de nuestro estudio podemos deducir que las arterias cubital y cubital recurrente aportan la vascularización dominante. Medialmente, el músculo recibe ramas de la arteria cubital y cubital recurrente, en la parte profunda del vientre muscular. Lateral y proximalmente, recibe ramas de la arteria mediana, mientras que lateral y distalmente recibe ramas de la arteria radial, que penetran en la superficie del músculo.

$$
\begin{aligned}
& \text { Palabras clave } \begin{array}{l}
\text { Estudio anatómico, Flexor digitorum } \\
\text { superficialis. }
\end{array} \\
& \text { Código numérico } \\
& 104-15830
\end{aligned}
$$

\begin{abstract}
We present an anatomical study that describes the distribution of the muscular perforators of the flexor digitorum superficialis muscle. In this study we dissected 15 forearms fixed according to Thiel method and coloured latex injection in the femoral vessels.The study was centered on the flexor digitorum superficialis muscle. Only muscular perforator arteries with diameters over $2 \mathrm{~mm}$ were selected. The vascular origin and length were also studied. In all cases, measurements were taken from the bicondyle line.

The total number of arteries obtained from the muscle belly was 219 , with the greatest percentage located in the half of the forearm. The principal vascular origin of the perforator arteries was the cubital artery.

From the results obtained in our work, we can deduce that the ulnar and the ulnar recurrent arteries are the most dominant supply of the muscle. Medially, it receives many large branches from the ulnar recurrent and the ulnar artery. Laterally and proximally, it receives small branches from the median artery, and distally, it receives several small branches from the radial artery that enter the superficial surface of the muscle.
\end{abstract}

\begin{tabular}{|ll|}
\hline Key words & $\begin{array}{l}\text { Anatomical study, Flexor digitorum } \\
\text { superficialis. }\end{array}$ \\
Numeral Code & $104-15830$
\end{tabular}




\section{Introducción}

Desde la introducción del concepto de angiosoma por Taylor y Palmer en 1987 (1), se han realizado varios estudios anatómicos de la vascularización del antebrazo aplicados al diseño y disección de nuevos colgajos basados en la distribución cutánea de los vasos perforantes (2). Sin embargo en nuestro conocimiento, no existen trabajos previos sobre el estudio macroscópico de las ramas arteriales que nutren el músculo flexor digitorum superficialis (FDS) : número de pedículos vasculares, longitud del trayecto libre, diámetro de los vasos y localización más frecuente.

El músculo flexor digitorum superficialis ha sido utilizado en Cirugía Reconstructiva principalmente como transferencia tendinosa en la parálisis radial (Boyes; Chuinard et al), para oponentoplastia en la parálisis baja del nervio mediano ( Royle-Thompson ; Bunnell) y en la reparación de la adducción del pulgar tras parálisis cubital.

Recientemente también se ha descrito la reconstrucción del aparato flexor profundo mediante transposición de un injerto tendinoso vascularizado libre o pediculado basado en ramas de la arteria cubital (3).

\section{Material y método}

Examinamos 15 antebrazos provenientes de 8 cadáveres por desarticulación glenohumeral, fijados mediante solución de Thiel (4) y coloreados mediante inyección de látex color en los vasos femorales. Realizamos un estudio del aporte vascular muscular desde su inserción ósea proximal en el epicóndilo medial, apófisis coronoides del músculo y cara anterior del radio, hasta la unión musculotendinosa.

\section{Técnicas de fijación e inyección}

Método de embalsamamiento de Thiel: La solución de embalsamamiento está compuesta por varios ingredientes con el objetivo de conservar la textura, volumen, color y forma del cuerpo, lo más parecidas posible a las condiciones normales.

La base fundamental de esta solución es una alta concentración en componentes salinos que causan la desnaturalización de las proteínas. Esta precipitación produce la homogeneización de los tejidos (5).

Tras infundir la solucion básica $(14.300 \mathrm{ml})$ y específica para cada tejido durante 3 días, los cadáveres son almacenados en contenedores con dicha solución durante al menos 6 meses.

Inyección de látex color: Se realiza en el departamento de Anatomía y Embriología Humana de la
Universidad Miguel Hernández, Alicante ( España), mediante inyección de $200 \mathrm{ml} / \mathrm{kg}$ de látex color (rojo) de forma automática tras canular los vasos femorales.

\section{Disección anatómica y mediciones}

Las disecciones de los 15 antebrazos se llevaron a cabo en el Aula de Anatomía de la Universidad de Medicina y Cirugía Miguel Hernández. Resecamos la piel hasta la fascia antebraquial a través de una incisión longitudinal desde epicóndilo medial a estiloides cubital y palma de la mano. Localizamos el compartimento anterior del antebrazo y comenzamos la disección desde su inserción proximal, separando los músculos braquiorradialis y flexor carpi radialis, localizando los vasos dependientes del eje radial y cubital. Realizamos mediciones en $\mathrm{mm}$ con un calibrador (Vernier Calipers $\left.{ }^{\circledR} 150 \times 0,05\right)$ del origen, diámetro y longitud de los pedículos vasculares del FDS (flexor digitorum superficialis) tomando como referencia la línea articular radiocarpiana.

\section{Resultados}

Realizamos la medición de las variables anteriormente descritas en los 15 antebrazos, realizando una distinción entre pedículos vasculares para el vientre muscular de FDS (flexor digitorum superficialis) y mesotendón (Fig. 1).

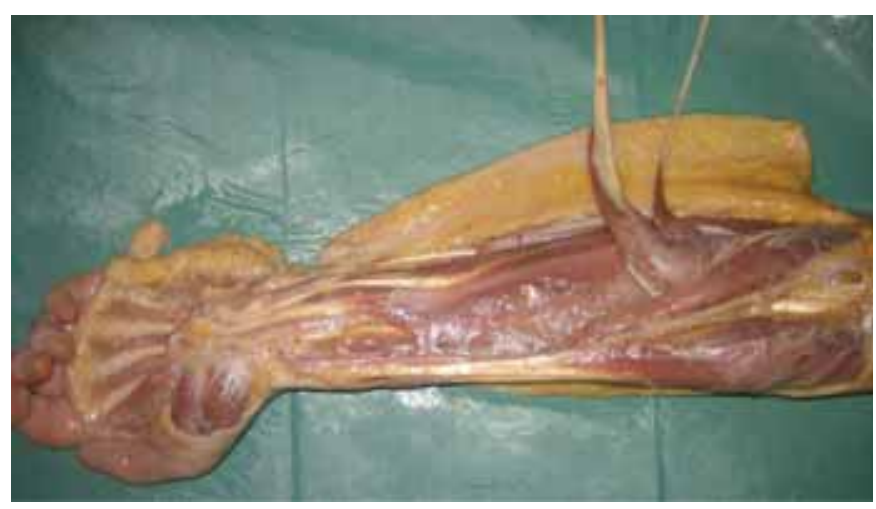

Fig. 1. Exposición del músculo Flexor Digitorum Superficialis (FDS).

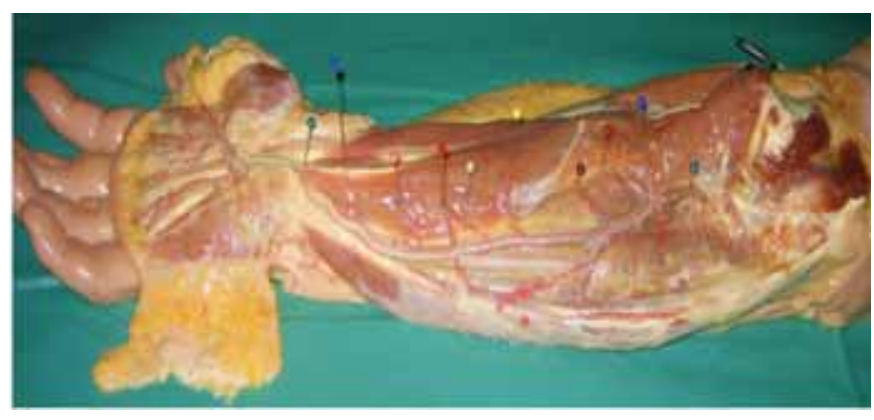

Fig. 2. Pedículos vasculares dependientes de la arteria cubital recurrente (Flecha). 


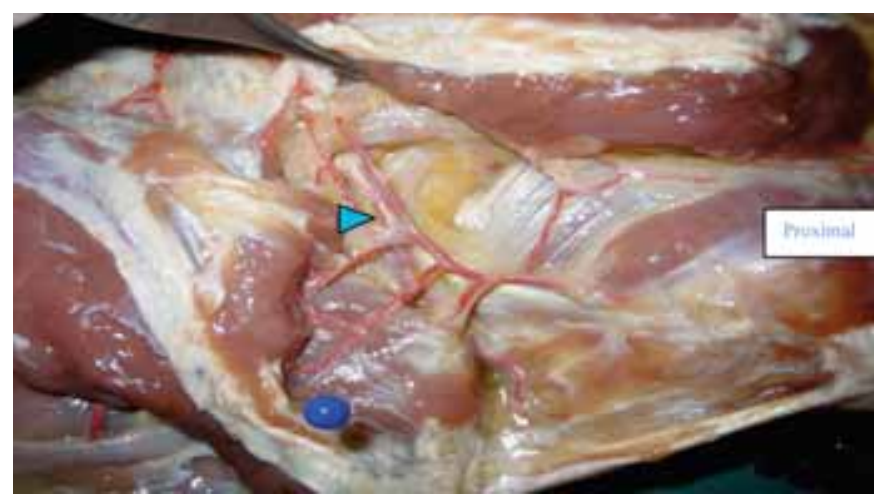

Fig. 3. Pedículos vasculares dependientes de la arteria cubital.

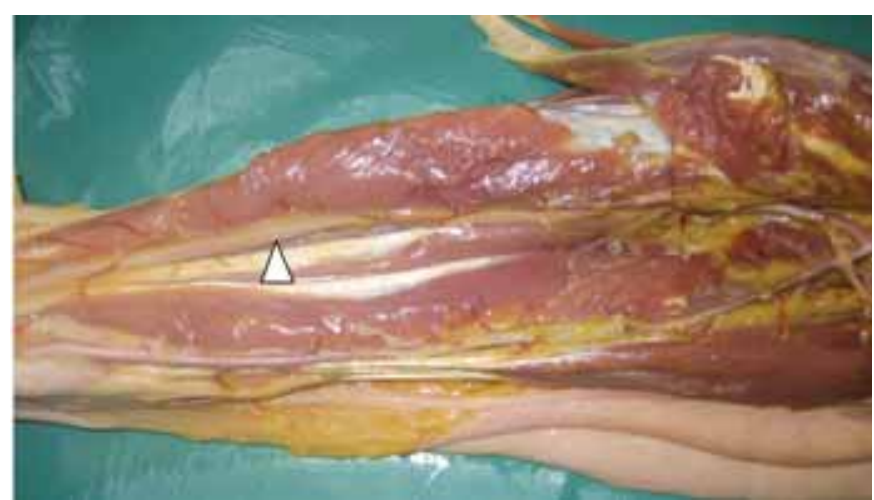

Fig. 4. Pedículos vasculares provenientes de la arteria comitante del nervio mediano.

Pedículos vasculares musculares: Identificamos un total de 237 pedículos vasculares (Desviación Estándar, SD: 6,143), con un diámetro medio de 2,23 $\mathrm{mm}(\mathrm{SD} 0,894)$ y un trayecto muscular libre medido desde el eje vascular principal de 4,291 mm (SD $8,54)$.

Arterias musculares axiales: Describimos 5 arterias axiales principales: arteria radial (Fig.2), arteria cubital , arteria cubital recurrente (Fig. 3), arteria interósea común y arteria interósea anterior (incluyendo la arteria comitante del nervio mediano) (Fig. 4). La distribución en porcentajes se expresa en el Gráfico 1.

En 1 de las 15 disecciones identificamos la arteria satélite del nervio mediano, rama de la arteria interósea anterior como principal donante de ramas vasculares para la región lateral del vientre muscular del FDS (Fig. 4).
aRADLAL ECUBITAL ONTEROSEAAAT IIITEROSEACOMUN aRECURRENTECUEITAL

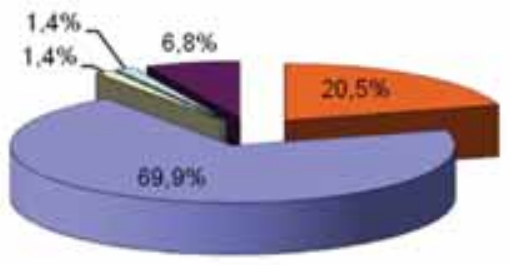

Frecuencias

Gráfico 1. Distribución en porcentajes de los principales ejes vasculares del músculo Flexor Digitorun Superficialis (FDS).

Para localizar los pedículos vasculares tomamos mediciones desde su emergencia en el eje vascular principal, hasta la línea articular radiocubital distal. Sin embargo, dado que la longitud del vientre muscular depende de la longitud del antebrazo en cada caso, decidimos convertir los valores absolutos adquiridos tras tomar como referencia la línea articular radiocubital distal, en valores relativos, dividiendo la distancia registrada para cada pedículo vascular entre la longitud total de cada antebrazo. Gracias a esta conversión pudimos localizar cada pedículo vascular como describimos en los Gráficos 2 y 3 , donde el 0 en el eje horizontal representa la estiloides radial/cubital y el 1 el epicóndilo medial/ lateral.

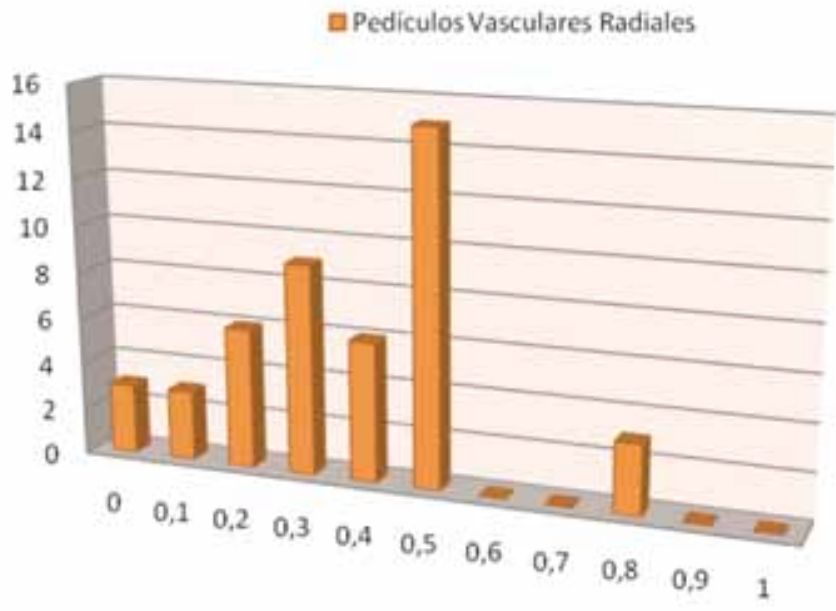

Gráfico 2. Distribución en valores relativos de los pedículos musculares dependientes de la arteria radial.

Observaciones: el eje horizontal representa la distancia entre la estiloides radial y el epicóndilo medial, medida en valor absoluto. El eje vertical representa el número de pedículos vasculares identificados a lo largo del vientre muscular.

Tabla I. Resumen de la vascularización del Flexor Digitorum Superficiales (FDS).

\begin{tabular}{lcc}
\hline & Pedículos vasculares musculares & Pedículos vasculares tendinosos \\
\hline RADIAL & 45 & 12 \\
CUBITAL & 153 & 27 \\
ARCO PALMAR SUPERFICIAL & & 24 \\
RECURRENTE CUBITAL & 15 & \\
INTERÓSEA COMÚN & 3 & 3 \\
INTERÓSEA ANTERIOR & 21 & 66 \\
TOTAL & 237 & \\
\hline
\end{tabular}




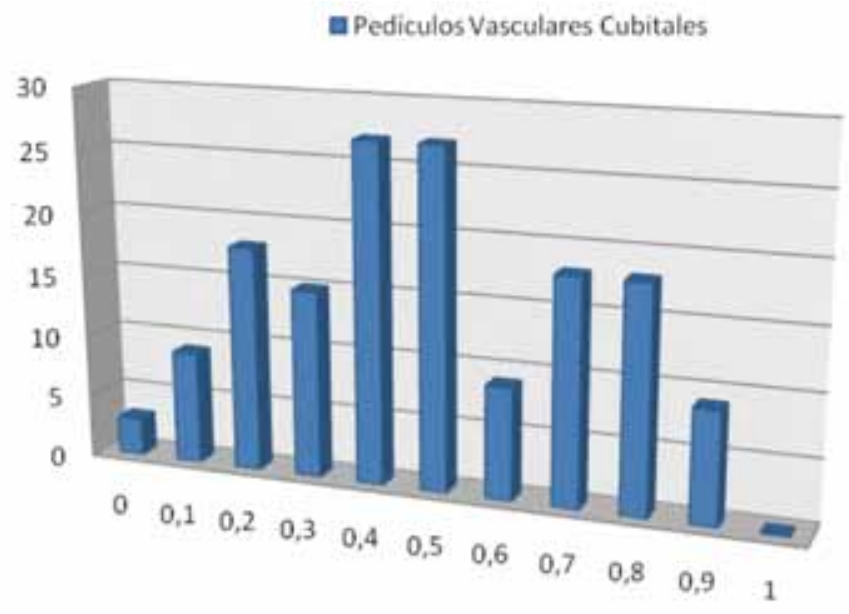

Gráfico 3. Distribución en valores relativos de los pedículos musculares dependientes de la arteria cubital.

Observaciones: el eje horizontal representa la distancia entre la estiloides cubital y el epicóndilo lateral, medida en valor absoluto.

El eje vertical representa el número de pedículos vasculares identificados a lo largo del vientre muscular.

El número total de pedículos vasculares identificados dependientes de la arteria radial fue de 45 (en los 15 antebrazos), localizados en su mayoría en el punto 0,5 (Gráfico 2).

El número de pedículos vasculares dependientes de la arteria cubital fue de 153 (en los 15 antebrazos), localizados en su mayoría en los puntos 0,4 y 0,5 .

Pedículos vasculares tendinosos: Identificamos 66 pedículos para la vascularización del mesotendón tras la disección de las zonas 3,4 y 5 de los antebrazos (Clasificación topográfica de la Federación Internacional de Sociedades de Cirugía de la Mano). Su diámetro era significativamente inferior al de los pedículos musculares, con calibre medio de $1,31 \mathrm{~mm}$ $(\mathrm{SD} 0,48)$ y longitud de trayecto libre $2,30 \mathrm{~mm}$ (SD $2,83)$.

Los principales ejes vasculares de origen fueron: arteria radial, arteria cubital (arco palmar superficial) (Fig. 5) y arteria interósea. En el Gráfico 4, se describe la distribución en porcentajes.

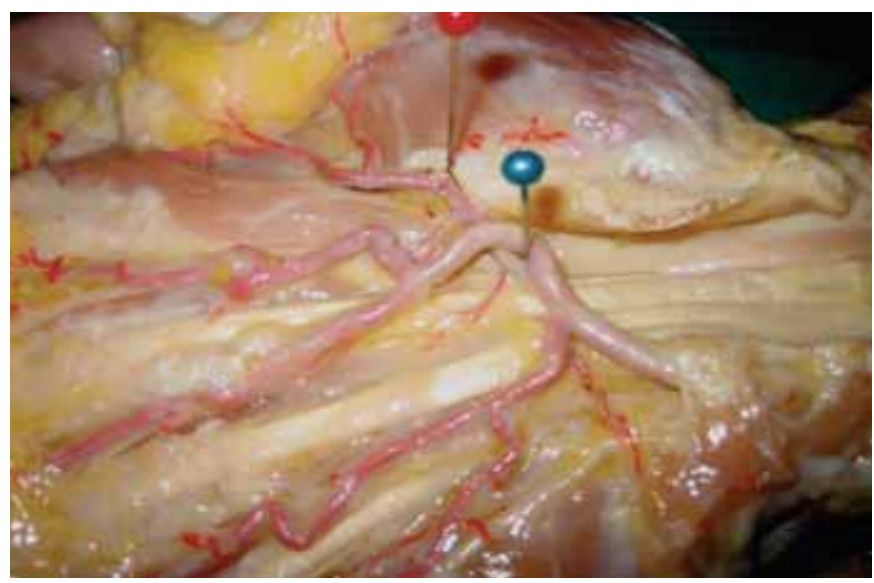

Fig. 5. Vascularización del mesotendón a partir de ramas del arco palmar superficial.

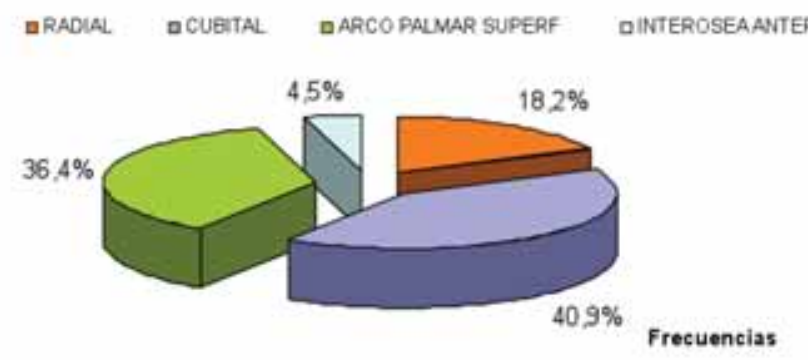

Gráfico 4. Distribución en porcentajes de los principales ejes vasculares para la vascularización tendinosa del músculo FDS.

En todos los casos identificamos una rama de la arteria cubital, proximal al canal de Guyon que vasculariza el mesotendón del FDS para cuarto y quinto dedos (Fig. 6), descrita por Guimberteau et al en 1993 (6), para la reconstrucción secundaria del aparato flexor, mediante transferencia del tendón del FDS del dedo anular vascularizado.

En la Tabla I presentamos el resumen de los pedículos vasculares disecados respecto a su origen.

\section{Discusión}

El músculo Flexor Digitorum Superficialis se clasifica, según Mathes y Nahai, siguiendo un patrón tipo IV con múltiples pedículos segmentarios. No encontramos en nuestro estudio contradicciones con los trabajos publicados anteriormente, aunque sí algunas diferencias $(1,6,7)$. Revol, M.P. en 1991 y posteriormente Inoue, Y. en 1996, describen la existencia de 3 angiosomas vascularizados por los siguientes ejes vasculares: arteria radial en la zona distal lateral, arteria comitante del nervio mediano lateral y proximal y arteria cubital en la región medial. En nuestras disecciones identificamos los mismos ejes vasculares axiales, con la excepción de la arteria comitante del nervio mediano, que sólo en una de las piezas se mostraba ampliamente desarrollada aportando más pedículos vasculares musculares que la arteria interósea anterior, donde se origina.

El principal aporte vascular para el músculo Flexor Digitorum Superficialis se origina en la arteria cubital

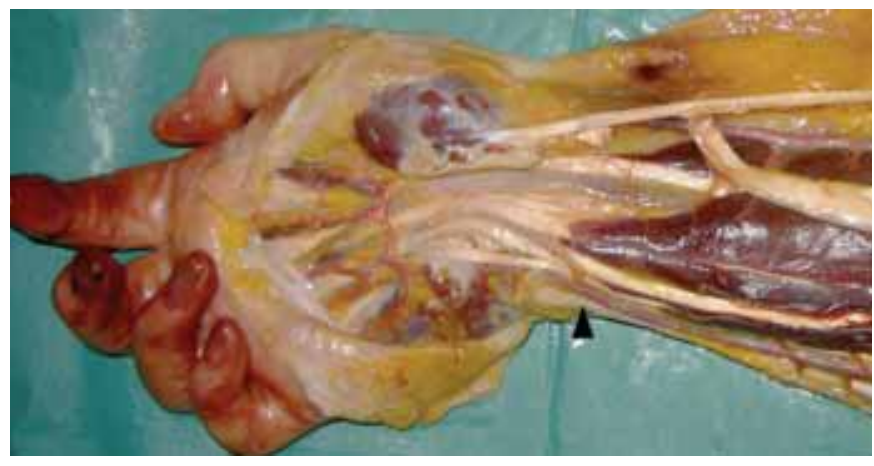

Fig. 6. Rama de la arteria cubital para la vascularización de la vaina sinovial común del carpo (Flecha). 
y cubital recurrente, datos confirmados por trabajos previos (1). El patrón vascular segmentario del FDS permite su disección en dos unidades funcionales, lateral y medial, basadas en la arteria radial y cubital respectivamente. En nuestro conocimiento, no hay trabajos publicados anteriormente que registren y describan la distribución de los pedículos vasculares del FDS, por lo que no es posible realizar un estudio comparativo respecto a otros autores.

De los resultados obtenidos podemos afirmar la existencia de pedículos vasculares provenientes de 3 ejes principales, con dominancia cubital en todos los casos y con la siguiente distribución en valores relativos, 0,4-0,5 / 1 para la arteria cubital y $0,5 / 1$ para la arteria radial.

Destacamos la presencia constante en todas las disecciones de 2 ramas de la arteria cubital, proximales al hueso pisiforme, que vascularizan la vaina sinovial común del carpo, descritas por Guimberteau et al en 1993 para la reconstrucción secundaria del aparato flexor, basado en la transferencia tendinosa superficial mesovascularizada (3). Esta técnica permite realizar la cirugía en un solo tiempo en casos complejos de reconstrucción tendinosa.

La necesidad de sacrificar la arteria cubital para transferir de forma vascularizada y pediculada los tendones de FDS, hizo perder popularidad a este técnica quirúrgica. Sin embargo, en 2003 Haerle, M. et al (6), describieron la dominancia radial, después de que la arteria cubital dé el tronco interóseo común, en el tercio distal de antebrazo y muñeca.Posteriormente, en 2006, Cavadas, P. y Mir, X. , modificaron la técnica qirúrgica permitiendo la transferencia libre de tendón vascularizado, conservando la arteria cubital, con excelentes resultados (8).

\section{Conclusiones}

Describimos 3 sistemas axiales y uno transverso en la vascularización del Músculo Flexor Digitorum Superficialis: la arteria radial, cubital, interósea anterior y cubital recurrente, respectivamente.

La distribución vascular segmentaria le confiere mayor viabilidad en situaciones de isquemia, así como la posibilidad de su disección longitudinal en dos unidades funcionales.

Las arterias cubital y cubital recurrente son dominantes en el aporte vascular, identificándose de manera constante 2 ramas cubitales proximales al canal de Guyon.

\section{Dirección del autor}

Dra. Nerea Comellas

Don Ramón de la Cruz 24. Madrid. España

e-mail: nereacomellas@hotmail.com

\section{Bibliografía}

1. Taylor, G.I. and Palmer J.H.: "The vascular territories (angiosomes) of the body: experimental study and clinical applications". $\mathrm{Br}$ J Plast Surg, 1987, 40(2): 113.

2. Inoue, Y. and Taylor GI: "The angiosomes of the forearm: anatomic study and clinical implications". Plast Reconstr Surg, 1996, 98(2): 195

3. Guimberteau J.C., Panconi B., Boileau R.: "Mesovascularized island flexor tendon: new concepts and techniques for flexor tendon salvage surgery". Plast Reconstr Surg, 1993, 92(5): 888.

4. Thiel, W.: "The preservation of the whole corpse with natural color". Ann Anat, 1992, 174(3): 185.

5. Wolff, K.D., et al.: "Thiel embalming technique: a valuable method for microvascular exercise and teaching of flap raising". Microsurgery, 2008, 28(4): 273.

6. Haerle, M., et al.: "Vascular dominance in the forearm". Plast Reconstr Surg, 2003, 111(6): 1891.

7. Parry S.W., Ward J.W., and Mathes S.J.: "Vascular anatomy of the upper extremity muscles". Plast Reconstr Surg, 1988, 81(3): 358.

8. Cavadas, P.C. and Mir X.: "Single-stage reconstruction of the flexor mechanism of the fingers with a free vascularized tendon flap: case report". J Reconstr Microsurg, 2006, 22(1): 37. 